\title{
Estimation of infection risk using symptoms of COVID-19: an approach based on fuzzy expert system
}

\author{
Serhat Ozbey $^{* 1}$ iD, Ahmet Koluman ${ }^{1}$ iD, Sezai Tokat ${ }^{2}$ iD
}

\author{
${ }^{1}$ Pamukkale University, Faculty of Technology, Department of Biomedical Engineering, 20160, Denizli, Turkey
}

${ }^{2}$ Pamukkale University, Faculty of Engineering, Department of Computer Engineering, 20160, Denizli, Turkey

\begin{abstract}
According to the published reports and studies, the symptoms of the disease caused by the COVID-19 virus have not yet been fully determined. It is a major stress on clinicians to make a correct and consistent decision about whether to apply the test or not, as many factors with extreme uncertainty need to be evaluated at once. In this study, it is aimed to provide assistance to the clinicians by processing the data using fuzzy logic based decision support system at the time of the decision-making process. In the designed fuzzy logic based decision support system, a fuzzy rule-base was created with linguistic information by interpreting the symptoms that are naturally uncertain by experts. With the help of the obtained fuzzy rule base, the input data of symptoms will be processed and the risk of a person being infected will be obtained as an output. As the results of the estimation module constructed with the existing parameters are examined, it is observed to be compatible with the data published before. In this context, a data set with 50 different patients were designed randomly to evaluate the system. For the analysis of the nonlinear mapping obtained with the Mamdani type fuzzy inference system, random test data is used and infection risk at rates varying between $12.5-83 \%$ was determined. The fuzzy logic based decision support system for COVID-19 can be accepted as applicable, flexible, and trustworthy for clinicians. It can be said that this system is not only suitable for COVID-19 but also applicable for future epidemics.
\end{abstract}

Keywords: COVID-19; decision support systems; epidemic; fuzzy expert systems; fuzzy logic

\section{Introduction}

Disease is often defined as any impairment, disorder or disability in the functions or physiological, biological structure of the human body (Hunter, 2009). It is diagnosed by physician's interpretation of characteristic signs and symptoms. In general, there are no clear standard case definitions for the diagnosis of a disease. Due to these uncertain signs and symptoms, the decision-making process for a physician becomes more complex. Clinicians try to interpret the patient's condition by requesting more tests in order to make more accurate decisions in the diagnosis process. The complex diagnostic process becomes more complicated by the increasing number of exams that need to be evaluated simultaneously. Computer-aided systems have been used to assist clinicians in this complex and uncertain disease diagnosis process. It is difficult to determine the nonlinear nature of the symptom, disease, and diagnostic process consisting of uncertainties with the two-valued classical logic. With its adaptation, learning, and reasoning capabilities, artificial intelligence (AI) began to play an increasingly important role in improving the quality of diagnostic effectiveness in healthcare. (Hossein et al., 2018). In our study, fuzzy logic as a class of AI method has been used to model the aforementioned complexities of the decision process.

Fuzzy logic is necessary in cases where the problem is complex to be solved and expert opinions are needed on this subject or it is difficult for individuals to evaluate and make decisions alone (Sen, 2009). Using linguistic expressions with

* Corresponding author.

E-mail address: sozbey151@ posta.pau.edu.tr (S. Ozbey).

https://doi.org/10.51753/flsrt.1010253 Author contributions

Received 22 October 2021; Accepted 15 December 2021

Available online 24 December 2021

2718-062X (C) 2021 This is an open access article published by Dergipark under the CC BY license. 
fuzzy logic, approximate results can be created in a shorter time without the need of a mathematical model (Novak et al., 2016). For instance, Akcam and Takada (2002) designed a fuzzy model to assist dentists in choosing the type of braces labelled as low, medium, and high in terms of pressure according to the mouth structure of the patients. It was observed that a significant part of the decisions made by the model were also approved by experts. Bates and Young (2003) developed a fuzzy model where mean arteriole blood pressure and urine excretion per hour turbid values were taken as inputs, and the amount of drug required to open the intravascular occlusion was estimated to control the intervention to return patients in intensive care units with stopped heartbeat. Stanley et al. (2003) used fuzzy logic to distinguish between malignant facial tumors and benign skin lesions for accurately diagnosing patients.

Grant and Naesh (2005) used fuzzy logic and decisionmaking systems in the field of anaesthesia where drug dosage and ventilation adjustment were aimed with variables such as respiratory rate and blood pressure. Benecchi (2006) designed a fuzzy model to assist clinicians in the diagnosis of prostate cancer. The intended use of the data presented in these studies and in the system designed in our study is similar. COVID-19, a multisystem infection with pneumonia, is the subject of this study. In their study, Pereira et al. (2004) developed a fuzzy model for the diagnosis of pneumonia for children by using $\mathrm{X}$ ray, shortness of breath and stethoscope auscultation, heart rate, body temperature, toxaemia and respiratory rate data. Data from 153 children who were previously diagnosed with pneumonia were used in the discriminant analysis and the designed fuzzy model. They reported that fuzzy logic has a $78.3 \%$ correct classification and is a good model for diagnosing diseases with its accuracy when compared with discriminant analysis (Pereira et al., 2004). Nascimento and Ortega (2002) designed a fuzzy model with a Mamdani inference engine to predict the risk of neonatal death in children within 28 days after birth where birth weight and birth age are taken as inputs and neonatal mortality risk was estimated as an output.

In COVID-19 rapid tests and screening tests, false positivity and false negativity rates are not stable. Also, the confidence interval is not clear. Therefore, wrong approaches may occur depending on the test results. At the same time, there may be exposure due to the testing process being carried out in hospitals. Within the telemedicine concept, it is obvious that people have less exposure during the diagnosis and treatment process without going to the hospital remotely. Cismondi et al. (2013) designed a fuzzy logic model with 11 variables as input and the output is used to estimate the patient's condition and to reduce the unnecessary laboratory tests during the treatment process of patients with gastrointestinal bleeding. An accuracy of more than $80 \%$ was achieved in the classification of necessary and unnecessary laboratory tests for patients. And thus, an average $50 \%$ reduction in the number of laboratory tests was obtained. Blackmore et al. (2011) stated that unnecessary radiological examination procedures have many negative consequences such as exposure to unnecessary radiation and waste of resources of health institutions. Therefore, when requesting a radiological examination, it was investigated whether it is possible to benefit from a decision support system that works integrated with electronic health record (EHR).

As of December 31, 2019, the World Health Organization issued a warning against COVID-19 pneumonia transmission of unknown origin in Wuhan, People's Republic of China. The virus was named as 2019 novel coronavirus (2019-nCoV) and was later named SARS-CoV-2 virus by the International Virus Taxonomy Committee (ICTV) in reference to its close similarity with SARS. SARS-CoV-2 is classified within the Betacoronavirus (subgenus Sarbecovirus) of the Coronavirus family. The virus binds to an angiotensin converting enzyme 2 (ACE2) receptor while inducing infection. SARS-CoV-2 is the seventh coronavirus $(\mathrm{HCoV})$ known to infect humans (Uras, 2021).

The clinical picture of COVID-19 can range from asymptomatic infection to severe disease, depending on age, gender and many other parameters. Mortality rates vary by country. At this time of the pandemic, early laboratory diagnosis of COVID-19 can aid clinical management and outbreak control. Diagnostic testing may involve detecting the virus itself (viral RNA or antigen) or the human immune response to the virus. Although there are differences in approach to the diagnosis of COVID-19 in different countries, as stated in the diagnosis documents reported by WHO, mainly, a patient is deemed to have a COVID-19 infection after evaluation of clinical symptoms by biosensor screening test, verification by PCR and subsequent computerized tomography image of the lungs (Genc, 2020; WHO, 2021).

In our study, the emergence of COVID-19 infection at varying age ranges, varying symptoms, and ambiguity due to changing practices in the test protocols were taken into account. Also, the symptoms of an infected person involved ambiguity in it. Using computer-aided systems to provide support in the decision-making process will provide more consistent and healthier results instead of leaving this decision, which is a process of evaluating many symptoms together, under human control. In this study, the most common symptoms of COVID19 infection were determined and the obtained data were included in a fuzzy inference system and the probability of a person's being infected that presents the symptoms was taken as an output.

\subsection{Fuzzy logic}

The concept of fuzzy logic gained meaning in our modern era with the article published by Lotfi A. Zadeh in 1965 who is considered as the modern founder of fuzzy logic (Zadeh, 1965). Fuzzy logic, which did not gain importance after the first published article, started to be used more in the mid-1970s. The articles (Zadeh, 1973; 1975) published by Lotfi A. Zadeh in those years, in which the applicability of fuzzy logic to complex systems were explained, had an effect on the increase of fuzzy logic researches. Since the 1980s, the numerous studies of the Japanese who have increased their interest in fuzzy logic have enabled fuzzy logic to reach its present level. Today, it is possible to use fuzzy logic in most conceivable areas (Shahbazova et al., 2020).

Fuzziness is all the incomplete and uncertain information that is possessed when there is no complete information about the subject being evaluated (Sen, 2009; Novak et al., 2016). The linguistic expressions used in daily life for variables are directly applied to label fuzzy sets and with infinite number of membership degrees and an inference mechanism a crisp output is obtained. With its linguistic elements, the human brain actually acts like a fuzzy logic system in decision making process. Likewise, a fuzzy logic is a system where many uncertain expressions are evaluated as input parameters and the result is reached after passing through fuzzy system processes (Shahbazova et al., 2020). 


\subsubsection{Fuzzy sets - classic sets}

In classical sets, an expression either belongs to one set or to another. On the other hand, in fuzzy sets, an expression may belong to one set to some extent, but also to other sets to different degrees. The difference of a fuzzy set and a classical set is shown in Fig. 1. As can be seen in the representative graphs, a situation in a classical set is either 1 or 0 . In a fuzzy set, instead of a sharp distinction, there are degrees of truth or falsehood of that situation. In classical logic, a proposition can be true or false. However, in fuzzy logic everything is a matter of degree (Zadeh, 1965; 1996).

The human life and linguistic information mostly involve vagueness that make it impossible to have sharp or precise distinctions in the world. Fuzzy sets and fuzzy logic theory provides us to use human expert knowledge and linguistic information (Zadeh, 1973; 1975; 1996).

\subsubsection{Fuzzy logic system structure}

The fuzzy logic system is based on linguistic information. It is based on finding the approximate non-linear mapping of a non-linear uncertain system input-output relation with a simple software process and without the need of mathematical system model. While developing a model for a fuzzy system, the input parameters of the problem are determined first. Then the input parameters are defined using fuzzy sets. Different membership functions can be used when defining fuzzy terms. Membership functions are explored in detail in (Zhao \& Bose, 2002). Output parameters corresponding to the inputs are also determined and defined using fuzzy terms. After that, the rule base is created with "IF-THEN" structure. In this process, a list of rules is created with verbal expressions based on expert knowledge about the problem. Fig. 2 shows the general structure of a fuzzy logic system.

In Fig. 2; the inputs are all the information (numerical and verbal) about the problem entries and the outputs are all the information (numerical and verbal) corresponding to the input parameters of the problem. The fuzzification block is the step of defining a precise input variable with the specified membership functions. Membership degrees of the input variable to sets are determined between 0 and 1 . At the end of this step, exact values are converted into fuzzy values (Kayacan \& Mojtaba, 2016). At the fuzzy rule base block, by using inputs and outputs, a rule list is created with the linguistic information of the expert knowledge in "IF-THEN" structure. The fuzzy inference system (FIS) engine works integrated with the rule base. At this step, all rules are evaluated separately and a decision is made for each fired rule. As a result, fuzzy output sets are obtained (Ross, 2004). The defuzzification is the last step in which the obtained fuzzy outputs are converted to a precise value. It is the opposite process of the fuzzification phase. Many methods are used in the defuzzification process. The most commonly used methods can be listed as: center of gravity, maximum average, weighted average, and area center (Ewald \& Mohammad, 2015).

\subsubsection{Mamdani fuzzy inference system}

FIS is also known as rule-based fuzzy systems, fuzzy expert systems, and fuzzy models (Sivanandam et al., 2007). FIS is the process of formulating the mapping from a given input to an output in which a decision is made based on information defined in the "IF-THEN" rule structure. In many areas, FIS can make predictions in decision-making processes (Liu, et al., 2019). In the literature, various FIS engines were developed that can solve specific problems better that the others. However, the two most popular methods in FIS can be listed as Mamdani and Takagi-Sugeno-Kang. Mamdani FIS engine is the most widely used inference method which is intuitive, suitable for human linguistic knowledge, and has an interpretable rule base (Mamdani \& Assilian, 1975). Mamdani FIS, as the output sets are also fuzzy, some operations need to be done later in this method. We can list all the operations of the method as: fuzzification, inferring from rules triggered on the rule base, merging and defuzzification after output clusters.

\subsubsection{Fuzzy rule base}

The fuzzy rule base was created in the form of an "IFTHEN" structure with linguistic information, using expert knowledge according to the considered symptoms and age data. When creating rules, the minimum method will be used for the AND operator and the maximum method will be used for the OR operator.

\subsubsection{Fuzzification}

Fuzzification is the calculation of membership degree of fuzzy sets to crisp input values. Triangular and trapezoidal membership functions are the most common types of membership functions as they are easier to represent (Pedrycz, 1994). Each rule is fired separately to a degree that is a function of the match between its antecedents and the crisp input.

\subsubsection{Aggregation}

In this study, for the composition of fuzzy relations MaxMin composition is used. It is necessary to collect all the triggered rules after the fuzzification process. Thus, the defuzzification process will be started for the sum of the fuzzy clusters formed as a result of all the collected rules. In the designed model, the maximum method is used for the combination process.

Thus, the largest of the smallest (max-min) method was used in the fuzzy model. The largest of the results obtained at the same input value will be taken as the basis for the defuzzification process. Representational graphics of fuzzification and defuzzification processes are shown in F. 3.

\subsubsection{Defuzzification}

At this stage of the FIS, a single crisp output will be obtained in the summation of all the triggered fuzzy clusters. The center of gravity (centroid), which is one of the most widely used defuzzification methods, was chosen. In this method the geometric center of gravity of the sum of the obtained fuzzy clusters is found. The crisp output obtained with center of gravity method can be given as follows:

$$
y_{\text {center }}=\int \frac{\mu(y i) y d y}{\mu(y) d y}
$$

\subsubsection{Decision support systems}

Human beings have to make decisions for the problems they face in most of their lives. Human decisions emerges as a 

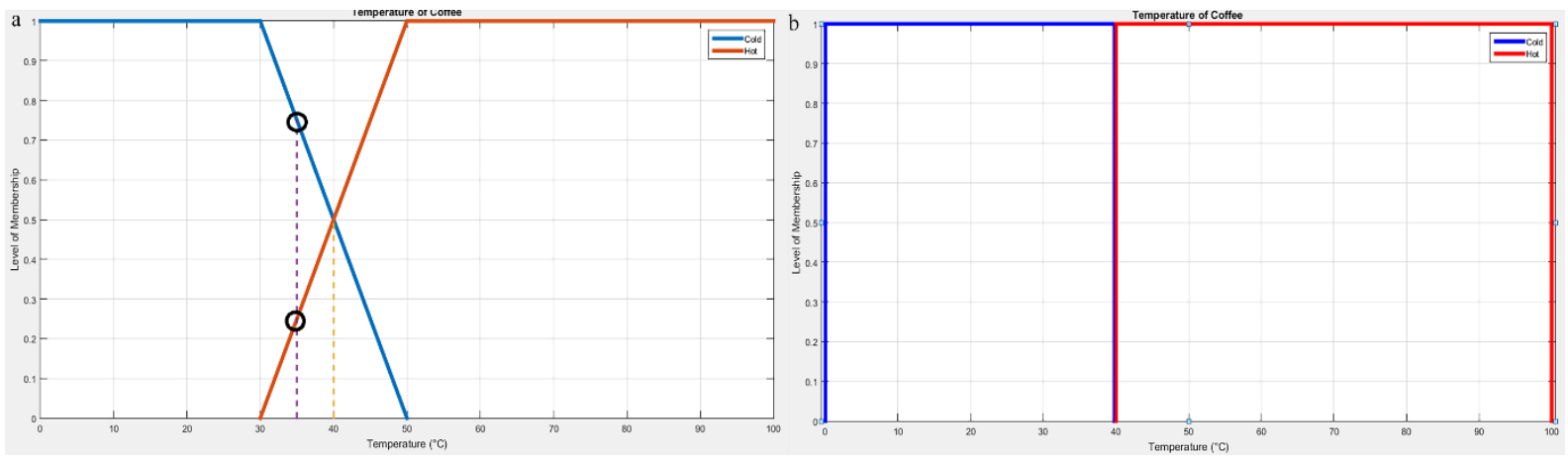

Fig. 1. (a) Fuzzy set representation plot. As an example, the value of $35^{\circ} \mathrm{C}$ belongs to the first set at a rate of $80 \%$, while $20 \%$ belongs to the second set, (b) Classical set representation plot. A certain value belongs either to the first set or to the second set.

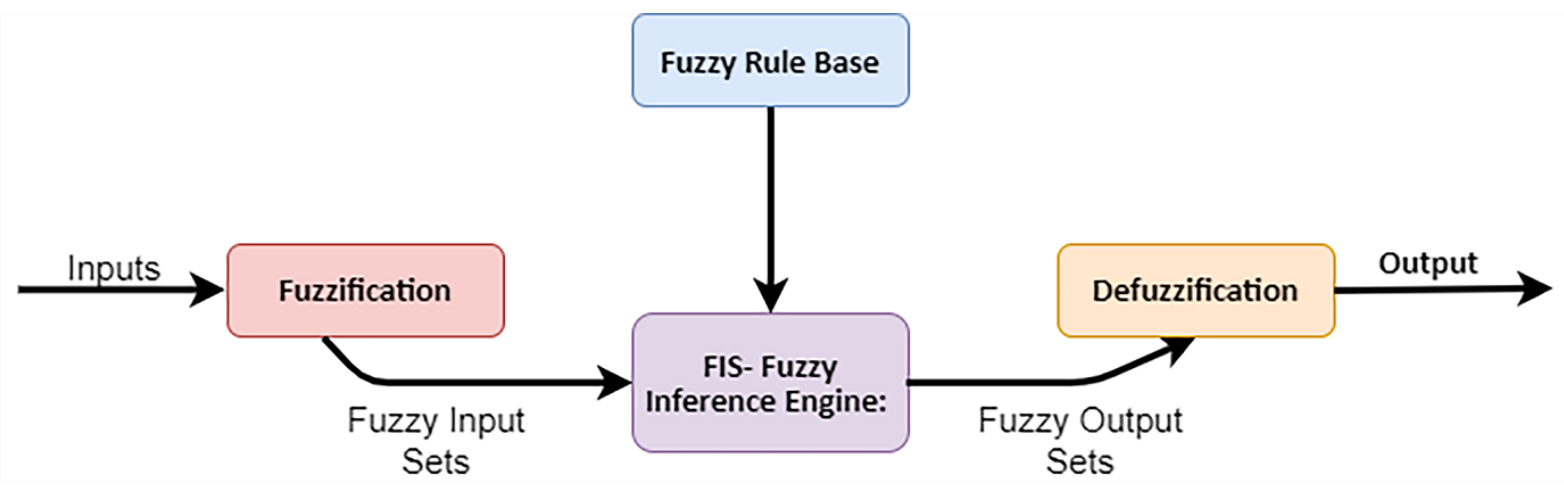

Fig. 2. General structure of fuzzy logic system.
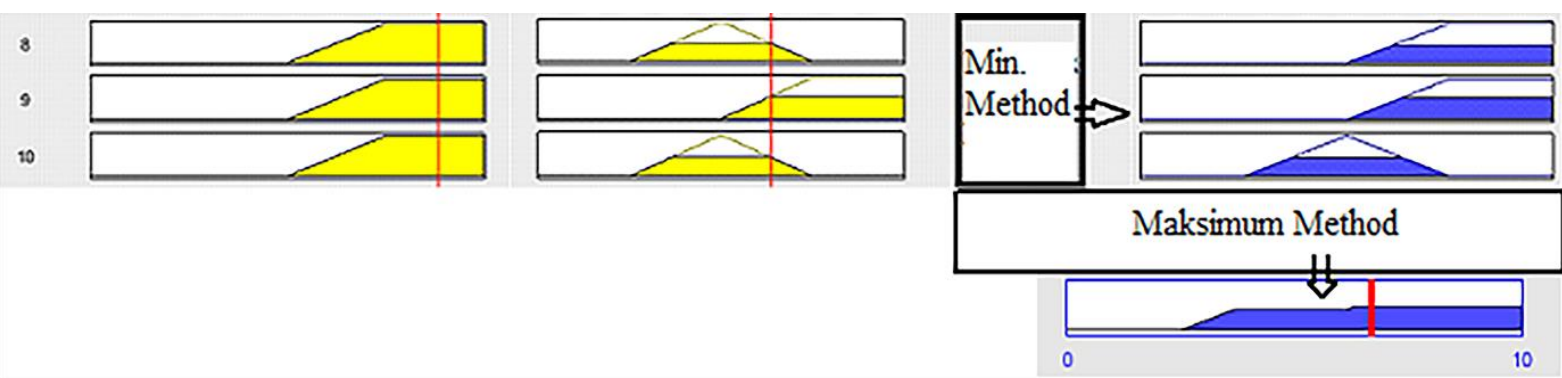

Fig. 3. A fuzzy system using the minimum ( $\min$ ) method for firing rules and using the largest (maximum) method for the combining process.

careful assessment of alternative choices in terms of the likelihood and value of the outcomes associated with these choices, and is always expected to end with a correct, true, right decision (Hickson \& Khemka, 2014). Many environmental factors and personality are effective in the decision-making and problem solving process.

Decision support systems are computer-aided systems that assist human decision makers by evaluating the whole data acquired in the complex decision-making process. As mentioned before, many factors are involved in the decision-making process and these factors direct the result. Decision support systems, on the other hand, provide selection forecasting and decision support to the decision-making mechanism by simultaneously evaluating environmental factors, similar choices previously experienced and all written, verbal, visual and audio data related to the problem. Decision support systems have emerged and developed in line with requirements (Musen et al., 2006).

Formerly, the developers of decision-making mechanisms had main difficulty in finding information about the problem. Presently, much more information about the problem can be reached, but the main problem now is to evaluate all data objectively and simultaneously. Decision support systems are user interactive systems for healthier, more consistent, accurate and effective decisions without ignoring the decision maker. It aims to assist the decision maker in accessing, summarizing, analyzing and concluding data (Miller et al., 1982; Musen et al., 2006).

\subsubsection{Clinical decision support systems}

Clinical decision support systems are computer-aided systems that assist healthcare administrators, doctors, nurses and experts in decision-making in their fields. The benefits of clinical decision support systems in the field of health are increasing progressively (Miller et al., 1982; Musen et al., 2006).

Clinical decision support systems offer many opportunities in the healthcare field (Kohli \& Piontek, 2008) and have begun to be used in many areas such as the management of increasing healthcare costs, determining the treatment services to be applied to the patient, laboratory management, hospital management, and interpretation of test results.

We can classify clinical decision support systems under 3 titles; (Musen et al., 2006).

a) Tools for knowledge management; Clinical decision support systems for information management in healthcare 
institutions enable the storage of information and data that will be needed by managers and clinicians and present the information to decision makers in a meaningful way at any time (Miller et al., 1982).

b) Systems for warning and focusing on one area; In such systems, the abnormalities are defined, and the user is notified of abnormal situations encountered. It also tries to prevent abnormal situations that arise from escaping the user's eye. For example, if a treatment is applied to the patient, the physicians will list the possible results regarding the treatment result. It informs the physician about the answers to the questions on how the drug may affect the patient's condition when drug therapy is applied. In general, it can be called a warning and reminder system (Miller et al., 1982; Musen et al., 2006).

c) Expert systems; in such systems, with the knowledge base created by the experts, patient data are processed and diagnosis or comments are made about the patient. Expert systems reduce the cost of services in the medical field or provide decision support in diagnosis and treatment processes. For instance, estimating the diagnosis of various diseases from the symptoms of the patient has been previously conducted with the Internist-1 project (Miller et al., 1982).

\section{Materials and methods}

In this study, the estimation of the risk of being infected according to the age and the most common symptoms during the COVID-19 epidemic, which the whole world is currently passing through, was made by using a fuzzy logic system having a Mamdani FIS.

The literature published in the determination of the most common symptoms of infected patients, as well as the reports of World Health Organization (WHO), Republic of Turkey Ministry of Health and other countries have been taken as a source (RTMH, 2021; WHO, 2021).

Symptoms and age data determined in the study were taken as an input for the fuzzy model and the probability of people being infected was taken as an output data. After the input and output data were determined, fuzzification was performed. Later, the probability of a person being infected is obtained by applying defuzzification to the fuzzy output of the fuzzy rule base and Mamdani FIS. The data processed according to Mamdani FIS, selected fuzzification and defuzzification methods were obtained. The designed fuzzy model was made with Matlab R2015a scientific package program and Fuzzy Logic Toolbox environment therein.

\subsection{Identification of symptoms}

The literature published while determining the symptoms of COVID-19 and the statements of WHO, Republic of Turkey Ministry of Health, US Department of Health and Human Services and the UK National Health Service were used (NHS, 2021; RTMH, 2021; WHO, 2021). Symptoms are broadly classified into main (common) symptoms, rare symptoms, and severe symptoms.
There are differences in the published literature and reports because the exact risk factors and severity of the symptoms are still unknown (Genc, 2020; Guan et al., 2020).

Since the COVID-19 outbreak has such an imprecise and vague expression, it was requested to evaluate the risk of infection with a fuzzy modeling. Generally, main symptoms are listed as fever, cough, and joint pain-fatigue. Rare symptoms include diarrhea, headache, loss of taste and smell. Shortness of breath is listed as a severe symptom (CDC, 2020; Guan et al., 2020; NHS, 2021; TRMH, 2021; WHO, 2021). In this study, main symptoms and the severe symptom listed above were decided to be used as an input data. The fever data, joint painfatigue level and shortness of breath of the person to be informed will be entered into the model as between 0 and 10 (0: very bad, 10: very good) and if there is a dry cough, it will be entered into the model as 1 , if not 0 .

The incidence of COVID-19 disease also varies according to age groups. According to the reports of World Health Organization and Republic of Turkey Ministry of Health (TRMH, 2021; WHO, 2021), it is seen that the majority of the infected patients are in the 25-49 age group.

According to the current reports of the Republic of Turkey Ministry of Health, $7.3 \%$ in children aged 15 and under, $13.7 \%$ between $15-24$ years old, $49.4 \%$ in the $25-49$ age group, $18.5 \%$ between the ages of $50-64,8.4 \%$ occurred in the $65-79$ age group, and $2.6 \%$ were 80 years old and above.

In this study, the risk of people being infected will be taken as the output data to be obtained as a result of the input data and the fuzzy rules. The risk of being infected is divided into 5 groups.

From symptoms entered as input data fever graph is shown in Fig. 4 (a), joint pain and fatigue graph (b), dry cough graph (c) and shortness of breath graph (d).

In the model made in the study, age data were examined in 4 groups to obtain more meaningful data. Age data groups are: $0-24,25-49,50-79,80+$. The age data graph created in the model is shown in Fig. 5.

Output data in the study were grouped as: very low, low, medium, high and very high. Triangle and trapezoidal membership functions are used when defining output data in the model. In the model, the risk of infection is given in percentage (\%). The graphic created with selected membership functions is shown in Fig. 6.

\subsection{Fuzzy rule base}

Some of the rules created by expert knowledge in the study are shown in Table 1. A total of 216 rules were created.

In the designed model, after entering the age of the person, the condition of joint-muscle pain, shortness of breath, the presence of cough and the fever level the risk of infection were taken as outputs. After a few data entries, the results of the model are shown in the Fig. 7.

The figure shows the rule viewer display, the data fired according to the input data, and the sum of the fuzzy rules combined with the clarification process.

Table 1

Examples of the rules created by expert knowledge.

\begin{tabular}{cccccc}
\hline Fever $\left({ }^{\circ} \mathbf{C}\right)$ & Age & Shortness of Breath & Dry Cough & Joint-Muscle Pain & Risk of Infection \\
\hline High & $80+$ & High & 0 (not presented) & Moderate & Medium \\
High & $80+$ & High & 0 (not presented) & Not Presented & Low \\
High & $80+$ & High & 0 (not presented) & Very Much & Very High \\
High & $80+$ & High & 0 (not presented) & Moderate & High \\
\hline
\end{tabular}




\section{Input data:}

Fever: 37.9 .

Age: 42.

Breath Condition: 7 (0: very bad to 10: very good).
Dry Cough: 1 (Yes) (0: not present, 1: present), Joint-Muscle Pain Condition: 6 (Between 0: very much and 10: not present).

Results: Person's risk of becoming infected: $74.9 \%$.

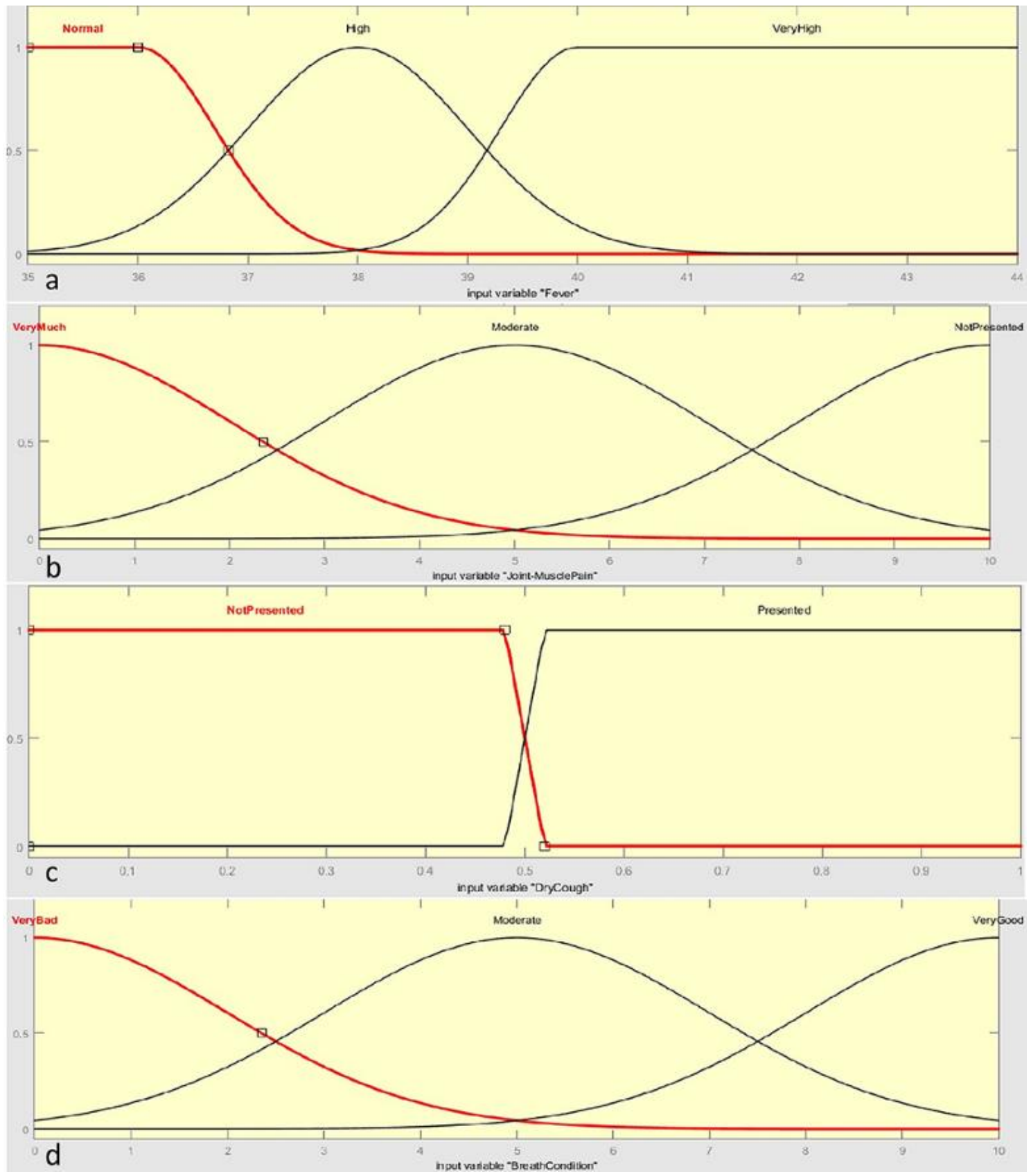

Fig. 4. Graphics of input variables. (a) Fever data, (b) Joint pain and fatigue, (c) Dry cough, (d) Shortness of breath.

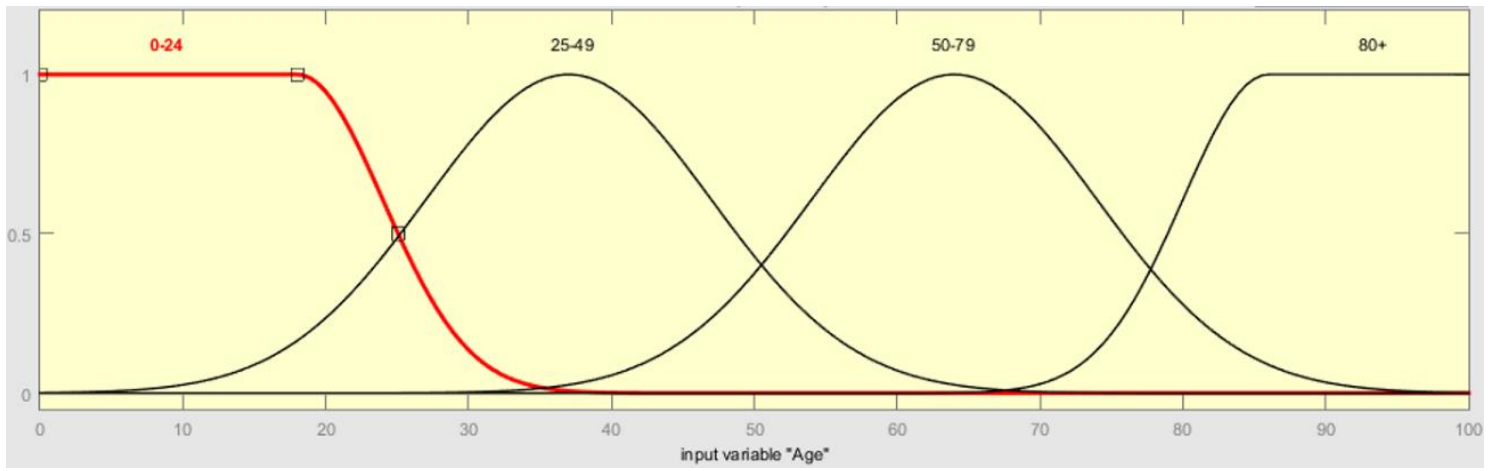

Fig. 5. Age data graphic. 


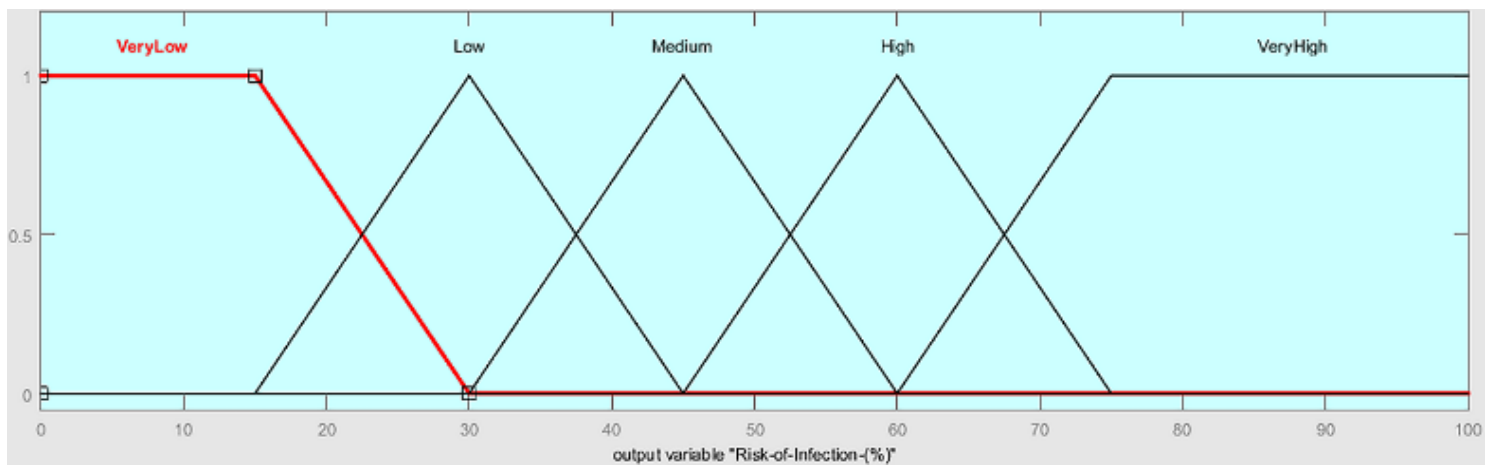

Fig. 6. Infection risk graphic as an output data.
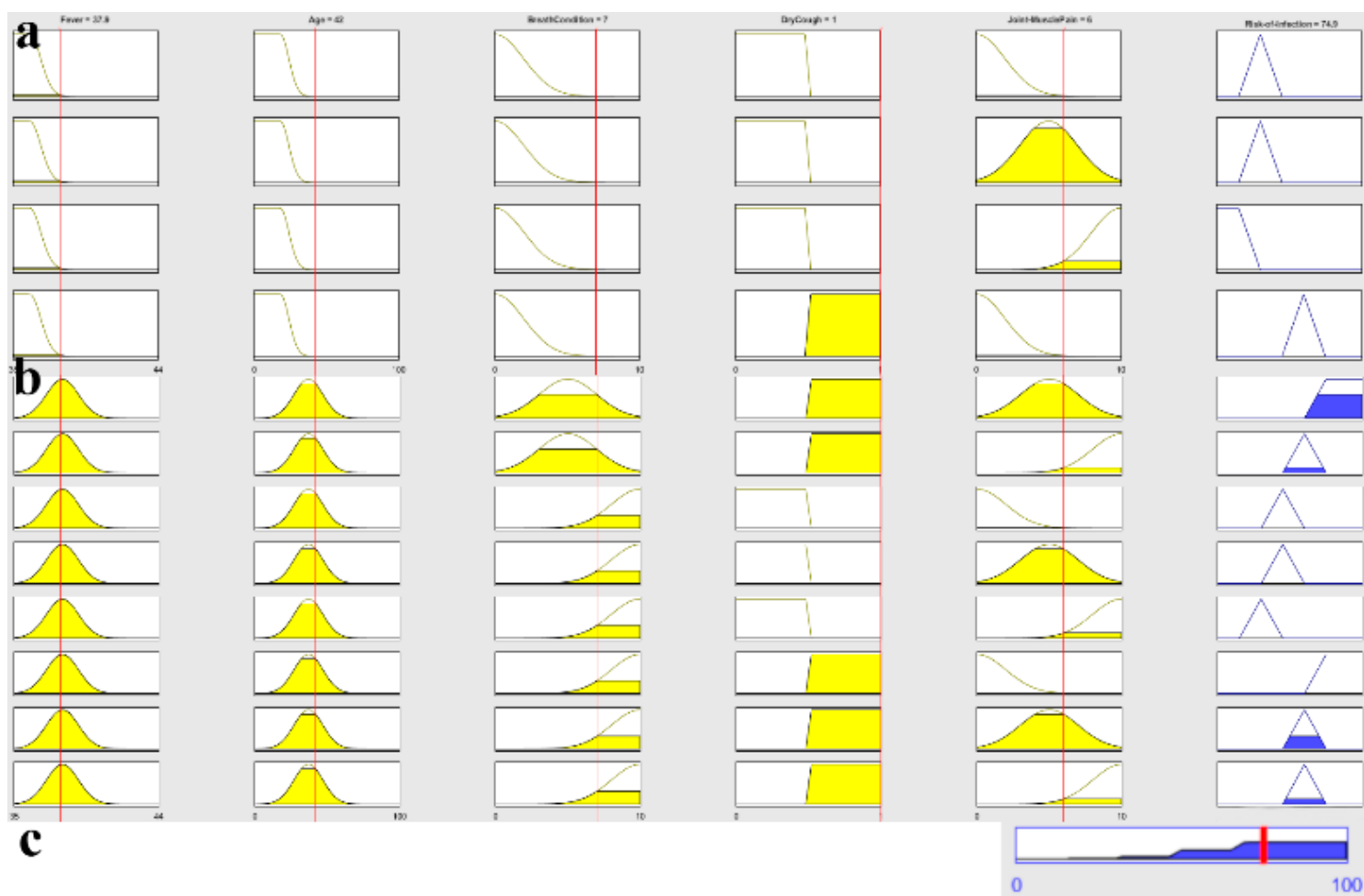

Fig. 7. Rule viewer display. (a) The rule viewer displays on the Fuzzy Logic Toolbox, (b) Some fired rules, (c) Results graphic after fuzzification and defuzzification processes.

\section{Results and discussion}

Outputs of the model are shown in Table 2. Model outputs as risk of infection are ranked (\%) from low to high.

$3 \mathrm{D}$ graphics of the designed model were drawn according to variables such as age-fever, age-shortness of breath, age-joint and muscle pain. 3D graphics created on Fuzzy Logic Toolbox are shown in Fig. 8, Fig. 9 and Fig. 10.

In this study, it was aimed to make predictions based on the symptoms of the disease by using fuzzy logic with the data of the pandemic COVID -19 that affected many people globally. In the first learning stage of the study, when the results of the estimation module constructed with the existing parameters in Fig. 4 a, b, c and d are examined, it is observed how the model will accommodate the estimate when taken from the real-life scenario.

Similarly, age data were observed to be compatible with the conducted studies and published hospital admissions. Model outputs as the percentage of infection were obtained by using a fuzzy rule base designed with expert knowledge and a proper defuzzification operation. In this context, 50 different patients set were formed depending on the experimentally applied data.
The fever, age, breathing condition, the presence of dry cough, joint and muscle pain belonging to these patients were added to these data sets and the risk of infection calculated as a result. As a result of randomly entered data with fuzzy logic created by us, infection risk at rates varying between $12.5-83 \%$ was determined. It is generally observed that the prediction deviation is less in studies conducted with fuzzy logic. In the fuzzy system designed by us, it is predicted that exposure and laboratory consumables will decrease, and it is in line with the studies mentioned in literature survey.

\section{Conclusion and recommendations}

There are almost no areas where software technology does not enter the human life. It is obvious that software support is required in order to ensure uniformity in the variations that occur in treatment approaches depending on the variability of experience despite the sample of information in the health sector.

Fuzzy logic makes it available to effectively deal with uncertainties in the decision-making process of electronic health records. In our study, a fuzzy logic based clinical decision. 
Table 2

The outputs of the fuzzy decision support system.

\begin{tabular}{|c|c|c|c|c|c|c|}
\hline \multicolumn{7}{|c|}{ Outputs of the Model } \\
\hline Data No. & Fever $\left({ }^{\circ} \mathbf{C}\right)$ & Age & Breath Condition & Dry Cough & Joint-Muscle Pain & Risk of Infection (\%) \\
\hline 1 & 36.0 & 8 & 10 & 0 (not presented) & 10 & 12.5 \\
\hline 2 & 36.1 & 18 & 10 & 0 (not presented) & 9 & 17.3 \\
\hline 3 & 35.9 & 77 & 10 & 0 (not presented) & 10 & 17.8 \\
\hline 4 & 36.5 & 23 & 8 & 0 (not presented) & 5 & 25.3 \\
\hline 5 & 39.2 & 13 & 9 & 0 (not presented) & 7 & 27.5 \\
\hline 6 & 37.5 & 21 & 10 & 0 (not presented) & 7 & 28.3 \\
\hline 7 & 39.0 & 14 & 8 & 0 (not presented) & 7 & 30.5 \\
\hline 8 & 36.2 & 41 & 6 & 0 (not presented) & 9 & 30.6 \\
\hline 9 & 36.8 & 71 & 7 & 0 (not presented) & 4 & 30.6 \\
\hline 10 & 37.0 & 49 & 4 & 0 (not presented) & 8 & 30.7 \\
\hline 11 & 36.6 & 28 & 4 & 0 (not presented) & 7 & 30.8 \\
\hline 12 & 38.3 & 86 & 9 & 0 (not presented) & 3 & 34.6 \\
\hline 13 & 38.9 & 28 & 8 & 0 (not presented) & 8 & 36.0 \\
\hline 14 & 38.1 & 32 & 7 & 0 (not presented) & 8 & 38.2 \\
\hline 15 & 36.7 & 35 & 9 & 0 (not presented) & 3 & 38.9 \\
\hline 16 & 36.8 & 57 & 4 & 0 (not presented) & 3 & 39.9 \\
\hline 17 & 37.0 & 30 & 8 & 0 (not presented) & 2 & 41.3 \\
\hline 18 & 35.9 & 77 & 7 & 1 (presented) & 9 & 41.3 \\
\hline 19 & 38.0 & 64 & 6 & 0 (not presented) & 6 & 42.2 \\
\hline 20 & 37.4 & 40 & 9 & 0 (not presented) & 4 & 43.3 \\
\hline 21 & 36.3 & 36 & 1 & 0 (not presented) & 2 & 43.5 \\
\hline 22 & 40.0 & 58 & 9 & 0 (not presented) & 6 & 44.6 \\
\hline 23 & 38.7 & 64 & 6 & 0 (not presented) & 4 & 45.0 \\
\hline 24 & 38.5 & 28 & 7 & 0 (not presented) & 3 & 45.9 \\
\hline 25 & 37.9 & 37 & 6 & 0 (not presented) & 4 & 46.1 \\
\hline 26 & 37.5 & 11 & 8 & 1 (presented) & 6 & 50.1 \\
\hline 27 & 36.8 & 26 & 6 & 1 (presented) & 9 & 50.9 \\
\hline 28 & 36.7 & 17 & 7 & 1 (presented) & 3 & 51.5 \\
\hline 29 & 37.9 & 35 & 6 & 0 (not presented) & 1 & 55.8 \\
\hline 30 & 38.3 & 26 & 3 & 1 (presented) & 9 & 58.9 \\
\hline 31 & 38.7 & 26 & 9 & 1 (presented) & 10 & 59.0 \\
\hline 32 & 37.1 & 61 & 2 & 1 (presented) & 4 & 60.4 \\
\hline 33 & 37.2 & 47 & 3 & 1 (presented) & 9 & 61.3 \\
\hline 34 & 37.3 & 37 & 2 & 1 (presented) & 9 & 62.0 \\
\hline 35 & 37.6 & 25 & 9 & 1 (presented) & 3 & 62.0 \\
\hline 36 & 39.6 & 53 & 7 & 1 (presented) & 9 & 65.0 \\
\hline 37 & 36.4 & 29 & 4 & 1 (presented) & 4 & 65.7 \\
\hline 38 & 39.6 & 67 & 8 & 1 (presented) & 7 & 66.0 \\
\hline 39 & 37.2 & 36 & 8 & 1 (presented) & 4 & 66.4 \\
\hline 40 & 39.9 & 70 & 8 & 1 (presented) & 8 & 67.8 \\
\hline 41 & 38.1 & 53 & 2 & 1 (presented) & 8 & 70.2 \\
\hline 42 & 39.5 & 82 & 3 & 1 (presented) & 4 & 70.8 \\
\hline 43 & 37.2 & 41 & 7 & 1 (presented) & 5 & 72.0 \\
\hline 44 & 39.8 & 57 & 6 & 1 (presented) & 7 & 74.7 \\
\hline 45 & 37.7 & 42 & 7 & 1 (presented) & 6 & 74.9 \\
\hline 46 & 38.7 & 43 & 6 & 1 (presented) & 7 & 75.1 \\
\hline 47 & 39.2 & 31 & 6 & 1 (presented) & 3 & 76.8 \\
\hline 48 & 38.9 & 29 & 3 & 1 (presented) & 5 & 78.1 \\
\hline 49 & 38.6 & 34 & 5 & 1 (presented) & 5 & 81.8 \\
\hline 50 & 40.0 & 37 & 1 & 1 (presented) & 1 & 83.0 \\
\hline
\end{tabular}



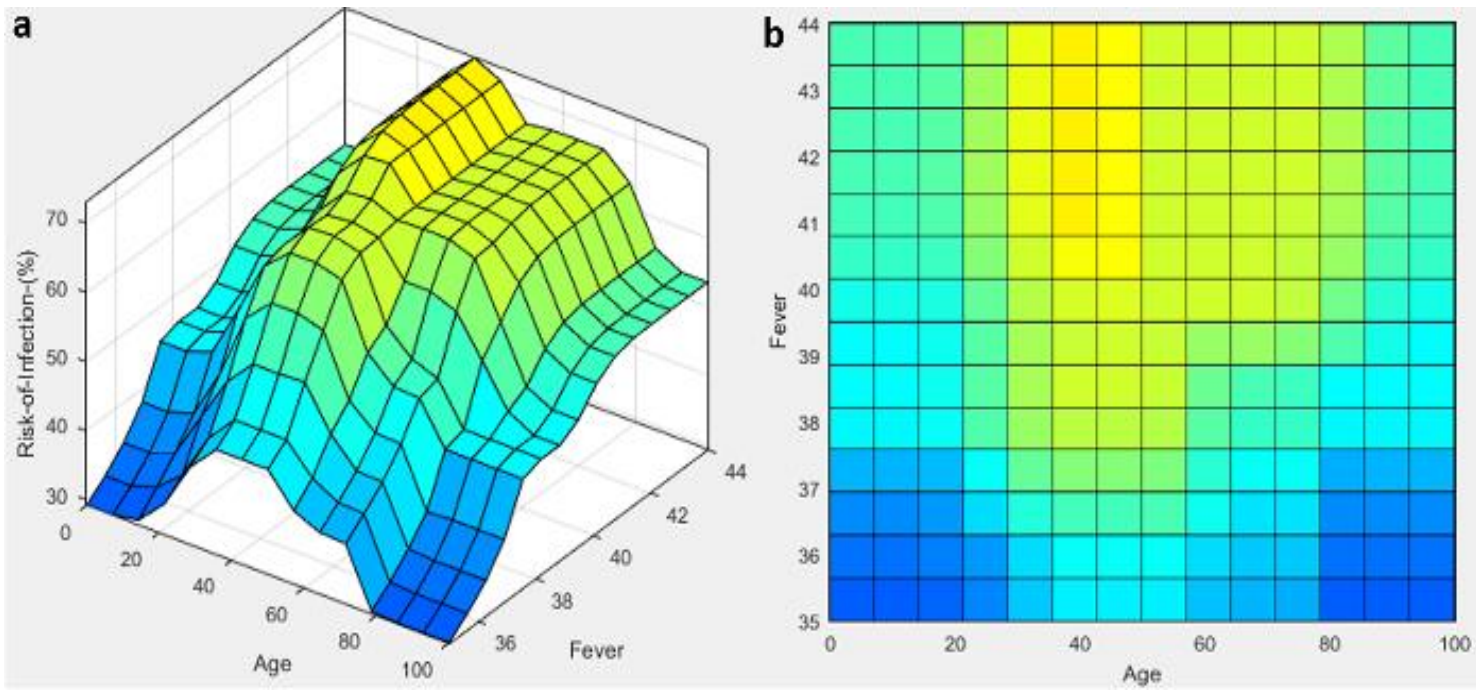

Fig. 8. (a) Output age-fever plot of the designed model, (b) Distribution of the age-fever according to the colored map.
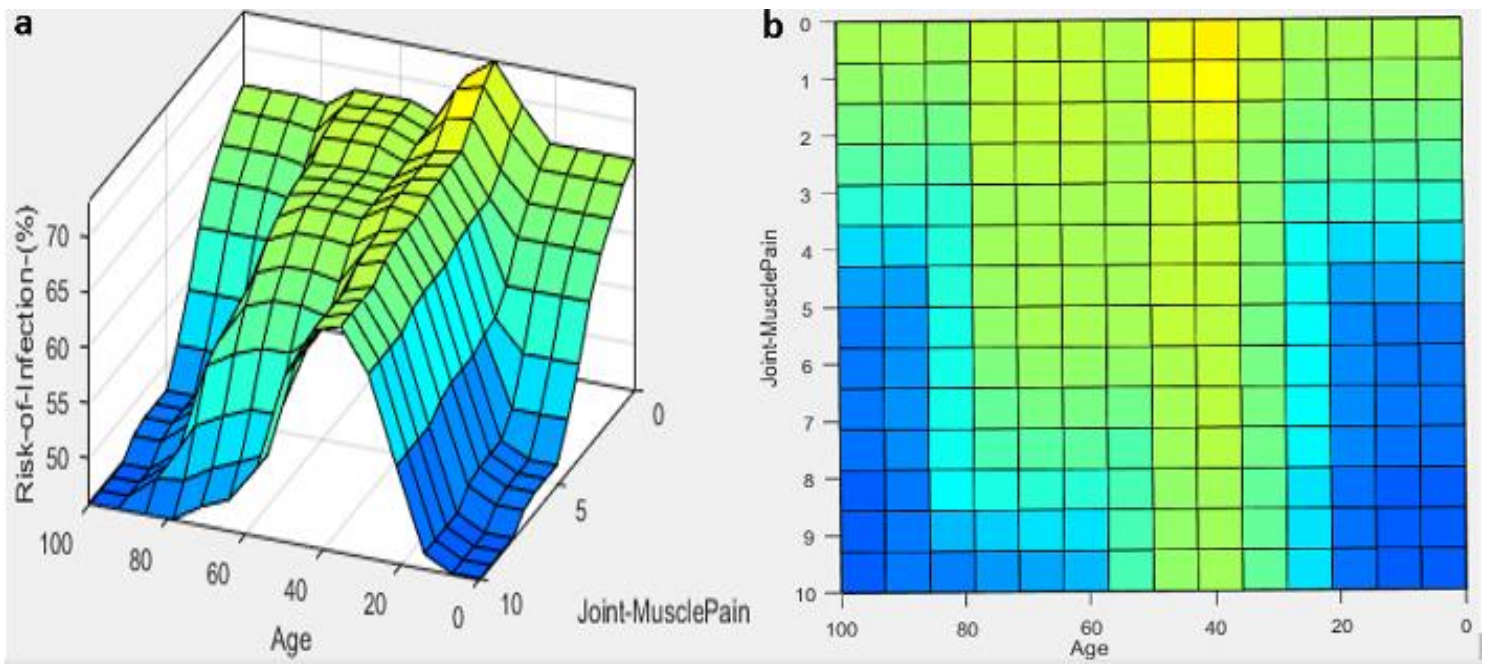

Fig. 9. (a) Output of the designed model, age-joint, muscle pain, (b) distribution of age-joint, muscle pain according to the colored map.
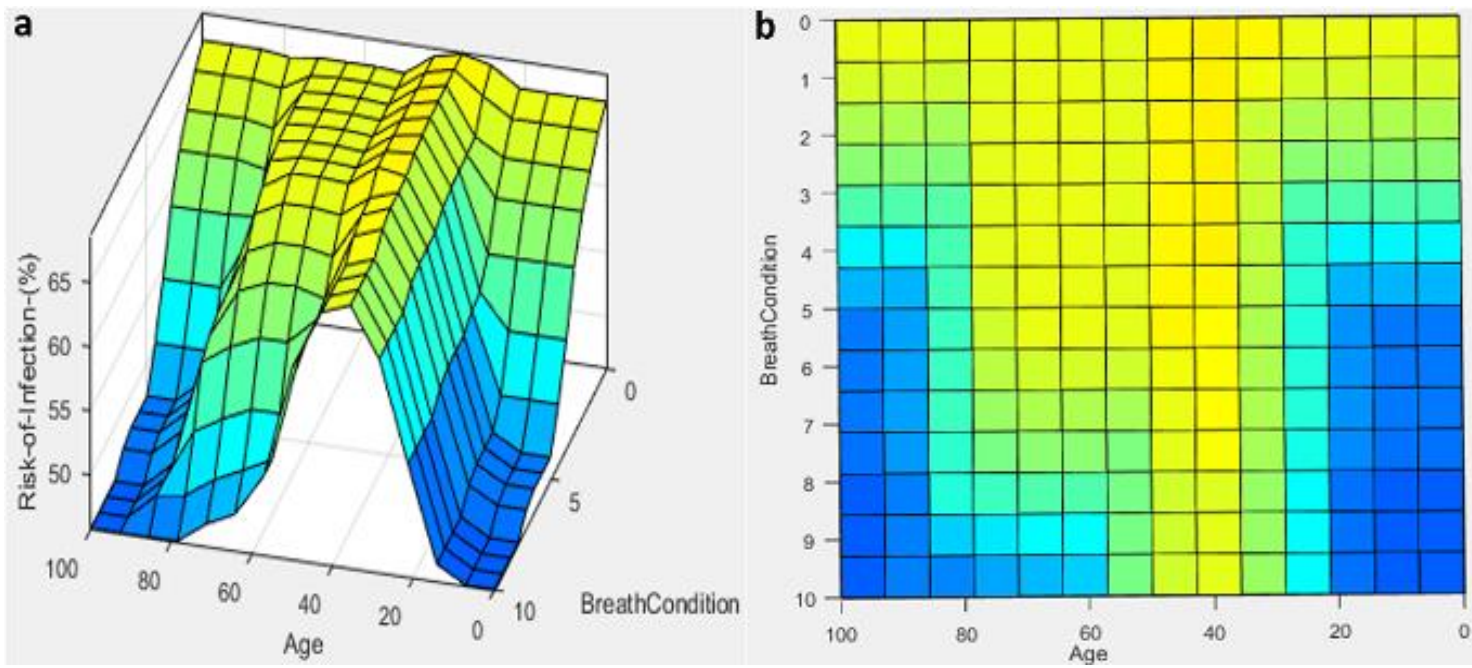

Fig. 10. (a) Output of the designed model, age-shortness of breath, (b) distribution of the age-shortness of breath according to the colored map.

support system is obtained as a pretest of COVID-19 for obtaining a risk scale to ensure that tests are directed to the right people.

It is difficult to break the resistance of the physicians related to artificial intelligence based decisions. However, the proposed fuzzy logic based system never replaces a physician; on the contrary, it is thought to contribute to the public health and health economy by supporting the physician's decisions. In addition to the rise of virtual communication in the COVID-19 pandemic, which occurred during the current decade and changed the world, it is observed that the effectiveness of software-supported prediction modules also became prominent 
and the concept of home healthcare services increased.

In addition to the insidious and changing symptoms of COVID-19, its fatal course continues without clear information about the public health risks. Considering the analysis of the fuzzy logic decision support system designed in this study, it is seen that positive results in estimation can be obtained using five different symptoms as input.

If new symptoms are decided to be appended to the proposed system, this can be easily obtained by adding new rules and revising the current rules to adapt to other symptoms. Therefore, the proposed system could support the clinicians and doctors in the diagnosis processes of COVID-19 by manually adapting as the disease change. When the results of our study are analyzed, it is observed that the related literature also has compatible results with our estimation results and that it is a

\section{References}

Akcam, M. O., \& Takada, K. (2002). Fuzzy modelling for selecting headgear types. The European Journal of Orthodontics, 24(1), 99-106.

Bates, J., \& Young, M. (2003). Applying fuzzy logic to medical decision making in the intensive care unit. American Journal of Respiratory and Critical Care Medicine, 167(7), 948-952.

Benecchi, L. (2006). Neuro-fuzzy systems for prostate cancer diagnosis. Urology, 68(2), 357-361.

Blackmore, C. C., Mecklenburg, R. S., \& Kaplan, G. S. (2011). Effectiveness of clinical decision support in controlling inappropriate imaging. Journal of the American College of Radiology, 8(1), 19-25.

CDC, (2020). United States of America Centers for Disease Control and Prevention. Retrieved from Centers for Disease Control and Prevention:https://web.archive.org/web/20200302201644/https://www .cdc.gov/coronavirus/2019-ncov/hcp/clinical-guidance-managementpatients.html , Last accessed on December 4, 2020

Cismondi, F., Celi, L. A., Fialho, A. S., Vieira, S. M., Reti, S. R., Sousa, J. M., \& Finkelstein, S. N. (2013). Reducing unnecessary lab testing in the ICU with Artificial Intelligence. Internal Journal of Medical Informatics, 82(5), 345-358.

Ewald, F., \& Mohammad, A. (2015). Optimal Placement and Sizing of Shunt Capacitor Banks in the Presence of Harmonics. In E. F. Mohammad A.S. Masoum, Power Quality in Power Systems and Electrical Machines (Second Edition) (pp. 887-959). Elsevier Inc.

Genc, B. N. (2020). Critical management of Covid-19 pandemic in Turkey. Frontiers in Life Sciences and Related Technologies, 1(2), 69-73.

Grant, P., \& Naesh, O. (2005). Fuzzy logic and decision-making in anaesthetics. Journal of Royal Society of Medicine, 98(1), 7-9.

Guan, W., \& et al. (2020). Clinical characteristics of Coronavirus. The New England Journal of Medicine, 382(18), 1708-1720.

Hickson, L., \& Khemka, I. (2014). Chapter Six - The Psychology of Decision Making, In Hodapp R.M. (eds) International Review of Research in Developmental Disabilities. Volume 47, (pp. 185-229), Academic Press.

Hossein, A., Gholamzadeh, M., \& Shahmoradi, L. (2018). Diseases diagnosis using fuzzy logic methods: A systematic and meta-analysis review. Computer Methods and Programs in Biomedicine, 161, 145172.

Hunter, L. E. (2009). The Processes of Life: An Introduction to Molecular Biology. MIT Press.

Kayacan, E., \& Mojtaba , A. (2016). Fundamentals of Type-1 Fuzzy Logic Theory. In M. A. Erdal KAYACAN, Fuzzy Neural Networks for Real Time Control Applications (pp. 13-24). Oxford: Elsevier.

Kohli, R., \& Piontek, F. (2008). DSS in Healthcare: Advances and Opportunities. In C. W. Frada Burstein, International Handbook on Information Systems (pp. 483-493). Springer.

Liu, K., Chen, Z., Wu, J., Tan, Y., Wang, L., Yan, Y., . . Long, J. (2019). "Big Medical Data Decision-Making Intelligent System Exploiting Fuzzy Inference Logic for Prostate Cancer in Developing Countries. IEEE Access, 7, 2348-2363.

Mamdani, E. H., \& Assilian, S. (1975). An experiment in linguistic synthesis with a fuzzy logic controller. International Journal of ManMachine Studies, 7(1), 1-13.

Miller, R., Pople Jr, H., \& Myers, J. (1982). INTERNIST-1: An experi- successful model for the diagnosis of infection.

This study was done by using a Mamdani-type fuzzy inference system which obtains a nonlinear mapping between the input values and the output. Thus, it has been tried to show that it is possible to predict COVID-19 with a Mamdani-type fuzzy logic-based decision support system. The results obtained against 50 randomly generated patient data were found to be successful by experts and doctors in this field.

Conflict of interest: The authors declare that they have no conflict of interests.

Informed consent: This manuscript did not involve human or animal participants; therefore informed consent was not collected.

mental computer - based diagnostic consultant for general internal medicine. New England Journal of Medicine, 307(8), 468-76.

Musen, M., Shahar, Y., \& Shortliffe, E. (2006). Clinical Decision-Support Systems. In: Shortliffe E.H., Cimino J.J. (eds) Biomedical Informatics. Health Informatics. (pp. 698-736). New York: Springer.

Nascimento, L., \& Ortega, N. (2002). Fuzzy linguistic model for evaluating the risk of neonatal death. Rev Saude Publica, 36(6), 686-692.

NHS, (2021). United Kingdom National Health Service, Symptoms of Coranavirus. Retrieved from NHS:

https://www.nhs.uk/conditions/coronavirus-covid-19/symptoms/, Last accessed on December 4, 2020.

Novak, V., Dvorák, A., \& Perfilieva, I. (2016). Insight into Fuzzy Modeling. John Wiley \& Sons.

Pedrycz, W. (1994). Why triangular membership functions?. Fuzzy Sets and Systems, 64(1), 21-30.

Pereira, J., Tonelli, P., Barros, L., \& Ortega, N. (2004). Clinical signs of pneumonia in children: association with and prediction of diagnosis by fuzzy sets theory. Brazilian Journal of Medical and Biological Research, 37(5), 701-709.

Ross. (2004). Fuzzy Logic with Engineering Applications. 2nd ed. West Sussex: John Wiley \& Sons Ltd.

Shahbazova, S. N., Sugeno, M., \& Kacprzyk, J. (Eds.). (2020). Recent Developments in Fuzzy Logic and Fuzzy Sets: Dedicated to Lotfi A. Zadeh (Vol. 391). Springer Nature.

Sen, Z. (2009). Bulanık Mantık Illkeleri ve Modelleme (Mühendislik ve Sosyal Bilimler), İstanbul: Su Vakfı Yayınları.

Sivanandam, S., Sumathi, S., \& Deepa, S. (2007). Introduction to Fuzzy Logic using MATLAB. Berlin: Springer.

Stanley, R., Moss, R., Van Stoecker, W., \& Aggarwal, C. (2003). A fuzzy based histogram analysis technique for skin lesion descrimination in dermatology clinical images. Computerized Medical Imaging and Graphics, 27(5), 387-396.

RTMH. (2021). Republic of Turkey Ministry of Health, COVID-19 Information Bulletin. Retrieved from https://covid19.saglik.gov.tr, Last accessed on October 7, 2021.

Uras, M. E. (2021). In silico comparative analysis of SARS-CoV-2 nucleocapsid $(\mathrm{N})$ protein using bioinformatics tools. Frontiers in Life Sciences and Related Technologies, 2(1), 1-9.

WHO. (2021). World Health Organization. Coronavirus disease 2019 (COVID-19) Situation Report - 89; Diagnostic testing for SARS-CoV2. Retrieved from https://www.who.int/health-topics/coronavirus, Last accessed on October 7, 2021.

Zadeh, L. (1965). Fuzzy set, Information and Control, 8(3), 338-353.

Zadeh, L. (1973). Outline of a new approach to the analysis of complex systems and decision processes. IEEE Transactions on Systems, man, and Cybernetics, 3(1), 28-44.

Zadeh, L. (1975). The concept of linguistic variable and its application to approximate reasoning. Information Sciences, 8(3), 199-249.

Zadeh, L.A. (1996). Fuzzy logic= computing with words, IEEE Trans. On Fuzzy Systems, 2, 103-111.

Zhao, J., \& Bose, B. (2002). Evaluation of membership functions for fuzzy logic controlled induction motor drive. 28th Annual Conference of the Industrial Electronics Society (pp. 229-234). Sevilla: IEEE. 
Cite as: Ozbey, S., Koluman, A., \& Tokat, S. (2021). Estimation of infection risk using symptoms of COVID-19: an approach based on fuzzy expert system. Front Life Sci RT, 2(3), 92-102. 